\title{
Computational intelligence in emotional or affective systems
}

\author{
Special Session of the 22nd Italian Workshop on Neural Networks (WIRN 2012)
} May 17-19, 2012, Vietri sul Mare, Salerno, Italy

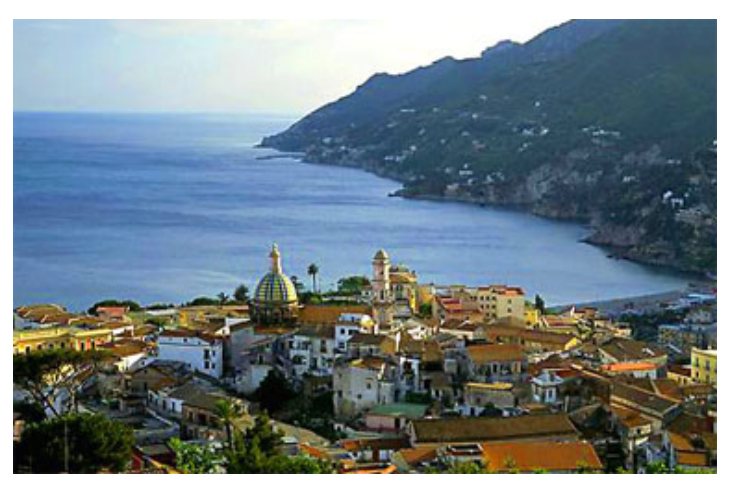

\section{Theme and scope of the session}

Computational Intelligence (CI) methods have shown great capabilities in modeling, prediction, and recognition tasks in DSP and a mature degree of understanding has been achieved in many application areas, in particular in complex multimodal systems supporting ambient human-machine or humanhuman interaction in smart environments, a field that is broadly addressed by the scientific communities and has a strong commercial impact.

At the same time, the emotional issue has recently gained increasing attention in Ambient Intelligence $(\mathrm{AmI})$ and smart environments due to its relevance in most common human tasks (like cognitive processes, perception, learning, communication and even "rational" decision-making) and, therefore, is highly relevant for the goal of human-like interaction with machines. The real challenge is taking advantage of the emotional characterization of humans to make the computer interfacing with them more natural and, hence, useful.
The scope of this session is to assess to what extent and how sophisticated computational intelligence tools developed so far might support the multidisciplinary research on the characterization of and ambient and system responses to human emotions and expression in interactive scenarios. Scientists working in the field are welcome to provide their contributions on the most recent trends, innovative approaches and future challenges. The session organizers believe to find in WIRN2012 a fruitful scientific scenario to deepen such issues.

\section{Topics}

Topics of the session include but are not limited to:

- Analysis and Identification of Human Emotional States;

- Application Oriented Redefinition of "Emotions";

- Computational Architectures for Affective Systems;

- Supervised and Unsupervised Learning Algorithms in Affective Systems;

- Emotional Human-Machine Interaction;

- Emotion Oriented Learning in Collaborative Knowledge Building;

- Ambient empathy.

The special session is organized inside the WIRN 2012 conference that is sponsored by the Italian Society of Neural Networks (SIREN, www.associazionesiren.org) in cooperation with the International Institute for Advanced Scientific Studies (IIASS, www.iiassvietri.it/), (Italy). 


\section{Important dates}

Contributions must be sent, no later than:

- March 252012 to: s.squartini@univpm.it, and iiass.annaesp@tin.it, phone/fax: +39071220 $4381 / 4453$ (ask for automatic confirmation of the reception);

- Notification of Acceptance: April 29 2012;

- Camera-ready copy: at workshop site, May 17 2012;

- Conference Dates: May 17-19 2012.

\section{Publications}

Accepted papers will be published by IOS Press, online on www.booksonline.iospress.nl/ and in print form, both in the Frontiers in Artificial Intelligence and Applications (www.frontiersinai.com) book series.

The maximum length of the full papers for contributors is 8 pages. Information on the paper format are on: http://www.associazionesiren.org/initiatives/.

\section{Registration fee}

The registration fee is 200 euros for the entire WIRN 2012 conference and include the social dinner. The registration can be done on-site in cash or through bank transfer (send an e-mail to Miss Tina Nappi, iiass.segreteria@tin.it, iiass.michele@libero. it; for bank coordinates).

\section{Invited speaker}

As in the previous WIRN editions there will be distinguished speakers for keynote talks. For this special sessions the invited speaker is Prof. Günther Palm, from Institute of Neural Information Processing, University of Ulm, 89069 Ulm, Germany.

Short Bio: Günther Palm was born in 1949. He studied mathematics at the Universities of Hamburg and Tübingen. After his graduation in mathematics (master in 1974, Ph.D. in 1975) he worked at the Max-Planck-Institute for Biological Cybernetics in Tübingen on the topics of nonlinear systems, associa- tive memory and brain theory. In 1983/84 he was a fellow at the Wissenschaftskolleg in Berlin. During spring-term 1987 he was research professor at the Technical University of Darmstadt. From 1988 to 1991 he was professor for theoretical brain research at the University of Düsseldorf. Since then he is professor for computer science and head of the Department of Neural Information Processing at the University of Ulm. From January 1997 to June 2000, he was the chairman of the Collaborative Research Center 527 on "Integration of symbolic and subsymbolic information processing in adaptive sensorimotor systems". From October 2000 to November 2002 he was dean of the School of Computer Science at the University of Ulm. He is working on information theory, pattern recognition, neural networks, brain models, associative memory and Hebbian cell assemblies.

Title talk: Towards a Redefinition of Emotions in Human-Computer Interaction.

\section{Organization}

\section{Program Co-Chairs}

Anna Esposito, Second University of Naples, Department of Psychology and with the International Institute for Advanced Scientific Studies (IIASS), Via G. Pellegrino 1984019 - Vietri sul Mare (SA), Italy, iiass.annaesp@tin.it; anna.esposito@unina2.it.

Stefano Squartini, 3MediaLabs, DII, Università Politecnica delle Marche, Via Brecce Bianche 31, 60131 Ancona, Italy, s.squartini@univpm.it, phone/ fax: +39071220 4381/4453.

Günther Palm, University of Ulm, Institute of Neural Information Processing, 89069 Ulm, Germany, guenther.palm@uni-ulm.de.

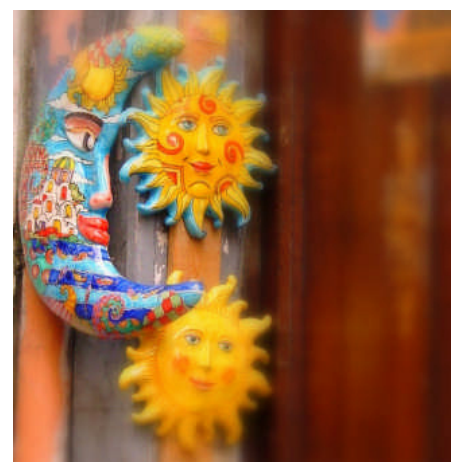

